doi: 10.2306/scienceasia1513-1874.2013.39S.095

\title{
Architectural framework for mobile multicast support in wireless mesh networks
}

\author{
Mistura L. Sanni ${ }^{\mathrm{a}}$, Aisha-Hassan A. Hashim ${ }^{\mathrm{a}, *}$, Wan Haslina Hassan ${ }^{\mathrm{b}}$ Farhat Anwar $^{\mathrm{a}}$, \\ Ahmed W. Naji ${ }^{\mathrm{a}}$, Gharib S.M. Ahmed ${ }^{\mathrm{c}}$ \\ a Electrical and Computer Engineering Department, International Islamic University Malaysia, Gombak, \\ 50728 Kuala Lumpur, Malaysia \\ b Department of Electronic Systems Engineering, Universiti Teknologi Malaysia, Jalan Semarak, \\ 54100 Kuala Lumpur, Malaysia \\ c Department of Science, International Islamic University Malaysia, Gombak, 50728 Kuala Lumpur, Malaysia
}

*Corresponding author, e-mail: aisha@iium.edu.my

Received 7 Jan 2013

Accepted 5 Apr 2013

\begin{abstract}
A wireless mesh network (WMN) is an infrastructure multihop ad hoc network with broadband capabilities. Multicasting is an efficient mechanism for delivery of group communication. However, the current IEEE 802.11s standard has no provision for efficient group communication delivery. Furthermore, the disruption in internet connectivity and loss of information when a mobile mesh client changes its point of attachment may lead to disruption in an ongoing session. The internet gateway (IGW), a prominent component, serves as the wired bridge between the mesh nodes and the internet, thus its placement determines the overall quality of communication in WMNs. Accordingly, a structural design for supporting multicast services in this architecture is indispensable. The main question this paper addresses is how multicast can be efficiently supported for mobile mesh clients in WMN architecture. This paper adopts the technique of employing multicast architecture to solve mobility problems for mobile mesh clients. Thus a framework of novel architecture for supporting multicast services for group application delivery over WMN is presented. The proposed architecture features enhanced functionality of IGW, shared multicast tree, optimal design for IGW and core placement, hierarchical mobility management, load balancing, and robustness. The overall objective is the provision of cost efficient WMN architecture while mobile multicast traffic delivery is supported optimally. This is the first effort reporting multicast service support framework for mobile mesh clients with a focus on IGW placement and functionality.
\end{abstract}

KEYWORDS: network architecture, gateway placement, multicast shared tree, optimization

\section{INTRODUCTION}

WMN is emerging as cost effective ad hoc broadband solution. Provision of multicast support in WMN is challenging ${ }^{1}$ and mobility management for mobile multicast source is even more challenging ${ }^{2,3}$. This is mainly due to the mix of wired and wireless interfaces/components of WMN and the backbone is essentially a wireless subnet. The generic architecture of WMN is hierarchical hybrid network consisting of IGW, wireless Mesh Routers (MR) and Mesh Clients (MC). The wired IGW is a prominent component, its placement and number deployed dictates the cost and performance obtainable in WMN. Incorporating multicasting involves derivation of a multicast tree which spans from multicast source(s) to every member of the multicast group ${ }^{4}$. Also the provision of mobility support as a design consideration is necessary for mobile MC to maintain connection during movement and even to initiate new connection while moving. The efficient mobile multicasting envisioned should hide the mobility of multicast senders and take cognizance of mesh connectivity specificities as a design consideration $^{5,6}$. These requirements thus call for a new architectural design for WMNs.

This architecture therefore should exploit the cost and performance benefits of optimized IGW placement ${ }^{7}$, together with scalable multicast routing framework such that the changes to multicast tree due to the movement of mobile multicast sender are addressed efficiently. A WMN is a MANET with infrastructure. However, the traffic characterization and architectural features in the two networks differ, thus solutions employed in MANET may not be efficient in WMN. Moreover, multicast tree construction and reconstruction is a major challenge in the provision of mobility management for multicast sender ${ }^{2}$, thus source based tree paradigm may not adapt efficiently in this sce- 
nario $^{7}$. In addition, the current IEEE 802.11s runs on the mandatory unicast Hybrid Wireless Mesh Protocol $(\text { HWMP })^{8}$. And existing research in WMN addresses mobile multicast without considering the effect of IGW placement ${ }^{9}$, while others considers IGW in the coordination of only multicasting, or only mobility management or security issues.

The proposed framework includes an extension of shared multicast tree for handling address changes for mobile multicast sender in the $(*, \mathrm{G})$ as against $(\mathrm{S}, \mathrm{G})$ in source-based trees. Also incorporated is the hierarchical mobility management because hierarchical architecture is natural to hybrid WMN (IGW layer, Relay or Mesh Router layer and Mesh Client layer) and such mobility management reduces excessive signalling by deploying different algorithms for micro and macro mobility. Apart from hierarchical scalable multicast architecture, robustness is also addressed by replicating the multicast traffic on all fixed multicast root. The approach employed in this novel architectural support for mobile multicast service in WMN is using multicast architecture to solve mobility problems.

There is no reported research that addresses IGW placement problems in the context of multicasting integrated with mobility management. The proposed solution is based on enhanced IGW functionality and multicast shared tree to produce mobile multicast compliant network architecture design that employs hierarchical flow model. Thus this paper is a proposal of new architectural framework addressing mobility management for group applications delivery via multicasting for mobile mesh clients.

The remaining sections of the paper are outline as follows: the next section analyses the mobile multicast problem and shows its significance; this is followed by discussions of previous related research works and framework for mobile multicast compliant architecture design. The remaining sections describe mobile multicast routing, conclusion and future works.

\section{MOBILE MULTICAST PROBLEM IN WIRELESS MESH NETWORKS}

The scenarios of close collaboration like real-time multimedia delivery require sending repetitive information to multiple recipients. Thus one of the possible efficient alternatives is to employ a restricted form of broadcasting known as multicasting. It is also crucial for these mobile devices to have access to the internet while moving. Hence WMN is appropriate as the access network because of its broadband capability and cost effectiveness. The transmission of real time multimedia data to a group of users is characterized in
Ref. 10:

(i) Multiple destinations which may not have fixed configurations.

(ii) Multimedia data constituents with delay constraints.

(iii) Large volume requiring higher bandwidth.

Given that the mesh clients with the hand-held devices can move about, it then becomes imperative to address the design issues of mobility support for such users. This is because mobility management is not a design consideration at the inception of multicast communication. Such mobility support is confronted by mobility challenges like IP address changes during handover, handoff latency, multicast tree instability, time constrained multimedia content and WMN specificities. Additionally, the visited network may not have multicast enabled routers. Into the bargain of multicast mobility management in WMN, an essential requirement is the consideration of IGW placement or selection and the coordination of mobility management within the subnetworks. Varying the placement or the positioning strategies implies a corresponding effect on the network topology which in turn affects handover latency and hence the overall throughput.

Multicast sender mobility problem also lends its own difficulty to the design of mobile multicasting in WMN. This arises from the need to maintain multicast tree when the source of multicast packets handover to another wireless mesh access point. Up until now, no standard has addressed the mobile multicast source problem ${ }^{11}$, even the task ahead of IETF multimob working group involves only multicast mobility for single hop architecture ${ }^{2}$. Therefore the instability of multicast tree due to mobility reason requires adequate research attention because the entire multicast session is affected as packets sent from such mobile multicast sender is not recognized by the corresponding multicast group receivers. Furthermore, the current IEEE 802.11s mandatory HWMP is a unicast Layer 2 forwarding mechanism that is based on MANET and proactive routing algorithms ${ }^{9}$. And unicast implementation cannot handle group application effectively ${ }^{12}$. Also network layer mechanism is required for globally distributed clients since IGW may not cope efficiently in coordinating MAC layer of distant nodes in the multihop architecture ${ }^{2,8,13}$.

This thereby necessitate research efforts on the provision of mobility support for multicast application delivery over internet as provided by WMN for scenarios including rescue operation in disaster areas, distance learning applications, mobile IPTV and other group content delivery applications. The notable research question is how can multicast service be 
efficiently supported for mobile mesh clients in WMN architecture? The proposed framework includes redefinition of the role of IGW, its selection and optimized placement, choice of source-based versus core based routing, hierarchical versus flat mobility architecture, placement of core router and path selection metrics. Consequently, the provision of this overall mobility support will handle the problem of multicast session breaks, tree construction instability, latency in handover and loss of packets.

\section{RELATED WORK}

The existing research addressing physical positioning and logical placements of IGW functionalities are based on the assumption of fixed positioning of IGW at the onset or by assigning the MC to nearest IGW ${ }^{14}$. However, these approaches did not put into consideration, the varying loads on the IGW and MR; and the specific requirements of the applications in view. Several other research address this crucial deployment problem and propose gateway placement and selection strategies.

In the functional placements scenarios, some research works extends the role of IGW with Mobility Anchor Point (MAP) function, while some incorporates multicasting capabilities or security mechanisms. In Ref. 15, MAP functionality is collocated in the IGWs, also Network Access Server together with Mobile Access Gateway are employed to address authentication, authorization and accounting, however this is implemented in Proxy Mobile IPv6 (PMIPv6) and it serves a security purpose on MANET ${ }^{16}$. proposes a central controller to maintain flow between IGW and other network elements, however another network element will expose the system to excessive signalling and delay. Even the suggestion of only one of the IGW as the network manager results in unfair loading and congestion. A detailed review of IGW placement optimization is presented in Ref. 17.

From the foregoing, none of these works integrated mobility management and multicasting into the placement and selection of IGW. Thus enhancing the functionality of IGW with mobility anchor and multicast functionalities may resolve the mobile multicast problem and under utilization of IGW and other resources in WMN. Also equipping this enhanced IGW with efficient load balancing technique and optimal selection algorithm may alleviate the bottleneck envisaged in the network.

Layer 3 multicast support is required for interworking for efficient utilization of network resources. Core Based Tree (CBT) multicast routing architecture proposed for routing on the internet ${ }^{18}$ implements a single shared tree for all sources in a multicast group irrespective of their number and location. Thus the approach reduces the rate of tree re-construction and ensures scalability. This shared tree is rooted at a designated router called core, however manual core placement is suggested in Ref. $18^{19}$. reports the significant effect of choice of designated routers on the performance of the CBT with respect to bandwidth, delay and traffic concentration. Thus core placement is an issue. Core placement method in Ref. 20 takes as input the distance vector information which does not involve the knowledge of the network topology ${ }^{21}$. proposes core tree optimization algorithm for maximizing the overall quality of real time P2P system. Core base tree multicast approach is proposed in Ref. 22 to address mobility, but in an IPv6 domain. And ${ }^{23}$ proposes distributed hierarchical multicast support in MANET. However, the network of choice in these research is not multihop WMN.

Hierarchical mobility management is proposed for MIPv6 to handle micromobility ${ }^{24}$ while $^{25}$ proposes M-HMIPv6 for the multicast implementation.

\section{MOBILE MULTICAST COMPLIANT ARCHITECTURE FRAMEWORK}

The problems identified in this work are addressed from the functional and physical network architectural perspective to guide the technical design of WMN architecture that is mobile multicast compliant (Fig. 1).

\section{Multicast support design}

Multicast communication is proposed for efficient delivery of group application especially multimedia content in real time, from multicast sender to multiple destinations over WMNs, so as to save scarce bandwidth and reduce the traffic on the network. Multicasting involves constructing an initial multicast tree which serves as the delivery tree between the multicast sender and the multicast receivers. The scenario as obtained in WMN is that mobile multicast senders can change their points of attachment to the network. When this occurs, the delivery tree constructed between the senders and the receiver group needs to be reconstructed to reflect the changes in the tree. The rate of reconstruction definitely grows as the number of times the sender changes location. The other alternative to these source-based tree approaches is to select a particular node to serve as static source or core router referred to as IGWCore to act for the multicast senders. Hence this problem is essentially a tree reconstruction necessitated by mobility.

The multicast problem described above needs to be addressed in order to support efficient multicasting 
Table 1 Characterization of core router and internet gateway.

\begin{tabular}{ll}
\hline Core router & internet gateway \\
\hline Motivation for CBT is for multicast scalability & IGW is mainly for interworking in WMN \\
Core routers are usually single node & Multiple IGW presentations \\
Possibility of additional core for robustness & Multiple IGW is for optimal coverage and load balancing \\
Core selection and placement are issues & IGW selection, placement and assignment are issues \\
Branches of core are non-core routers & Branches of IGW are relay nodes \\
\hline
\end{tabular}

\section{$\diamond$ Mesh Client \\ -... Wireless link \\ — Wired link \\ IGWCore
Mesh Router}

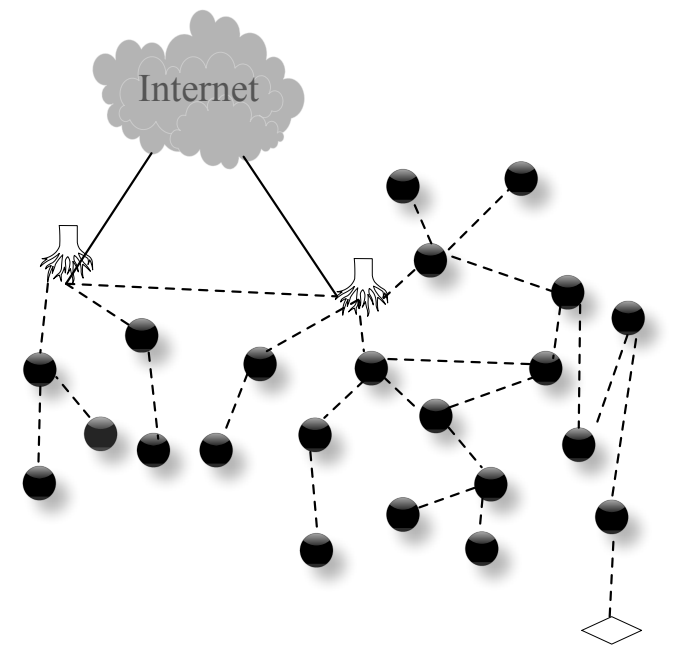

Fig. 1 Proposed mobile multicast network architecture framework.

in WMN. Scalability and optimized placement of core router is envisioned in the multicast support approach proposed in this work. Hence, this proposal exploits the core node strategy of CBT architecture for multicast support in WMN which incidentally also serves as a solution for the mobile multicast sender problem, since the core is static irrespective of the location of the multicast sender. However, the CBT architecture and the WMN architecture require some modifications for this implementation. For instance, the main motivation for CBT is multicast scalability while the core router framework in WMN is proposed with a view to handle mobility management and multicast support for mobile and active senders in a
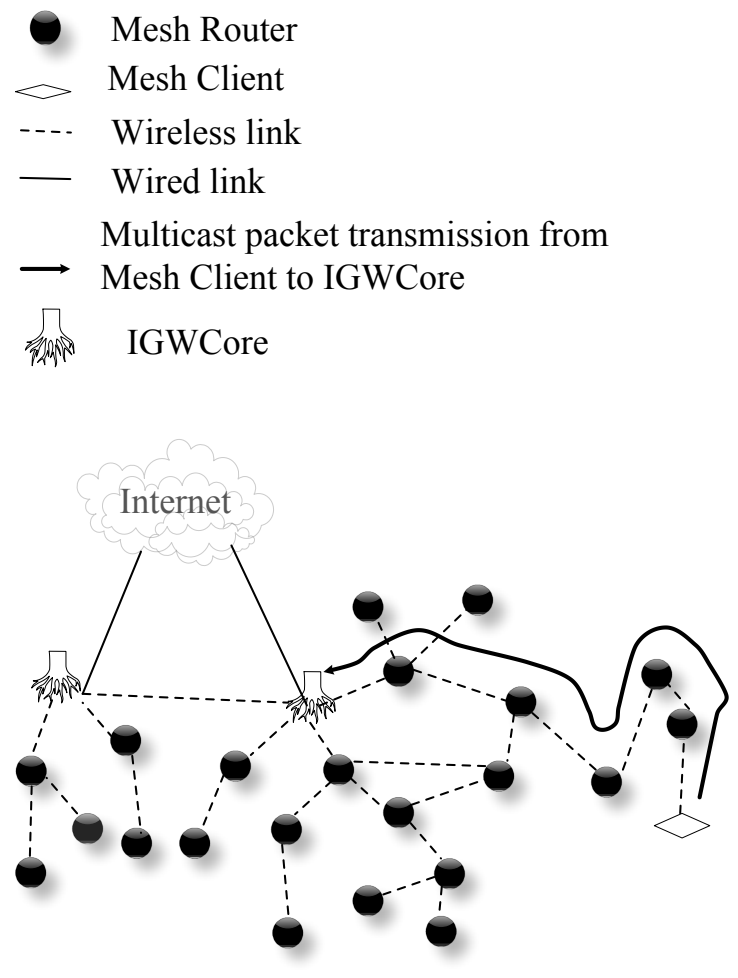

Fig. 2 Multicast sender discovers the nearest IGWCore.

multicast communication. The CBT architecture and the eventual incorporation in $\mathrm{WMN}$ are characterized in Table 1.

In Ref. 18, multicasting based on CBT implementation employs single shared tree paradigm to span all the group receivers. This is an appropriate choice because CBT is scalable and has routing independence:

(i) Global information maintenance on the location of multicast group members is required for the computation of shortest path by each router, this results in scalability problems with respect to processing and maintenance cost

(ii) CBT tree is stable with respect to changing location of multicast sender

Thus the multicast sender is strictly not partic- 
ipating in the multicasting as it implements unicast communication to send packet to the core, thus only group members and the core require the knowledge of the multicast $\operatorname{tree}^{18}$ (Fig. 2).

Consequently, the IGWCore router needs to be identified, its selection and optimized placement is an important task in this proposed framework.

\section{Enhanced IGW functionality and optimized placement}

IGW is a prominent component in WMN, the proposed architecture is an enhancement to the generic hybrid WMN architecture. The IGW only serves the bridge function for Interworking in the generic WMN. Essentially, the first premise is a multicast enabled router being deployed as a core router, that is, multicast root. This core router functionality is incorporated on IGW. Hence internet connectivity function in WMN and the static multicast root functionalities now forms the new enhanced functionality of IGW referred to in this proposal as IGWCore. The placement of Core router is shown to have significant effect on core-based tree routing, so also is the significant cost-performance implication of placement of IGW in WMN. Hence the IGWCore placement optimization is considered in this framework with the objective of determining its number and placement such that the MR-IGW path length, depth of the tree, the number being deployed are all minimized while the requirement for delivery of mobile multicast traffic is satisfied. The formulation of this problem is presented in Ref. 17 as a capacitated routing problem (CRP).

\section{Hierarchical mobility management design}

Hierarchical mobility management is proposed for the architecture to partition the mobility traffic, and to also employ IGW to perform as mobility anchor point. The IETF HMIPv6 concept is enhanced to reduce the excessive signalling overhead in the processing of binding updates. There are provisions for varying degrees of movement of mobile sources which includes those associated with the following movement scenarios:

(i) within the coverage of initial MR

(ii) outside the coverage of initial MR and IGWCore

(iii) outside the coverage of initial MR but within same IGWCore

Cases (i) and (iii) are defined as local or micromobility while case ii is defined as macro mobility and thus handled with different algorithm. Thus IGW in WMN is proposed to serve as the mobility portal. Hence, the coverage algorithm of mobile anchor placement and the selection and optimization algorithm of IGWCore for the deployment of mobile multicast application are implemented as an integrated concept at a single point.

The functionality of IGW is enhanced for multicast and mobility management reasons so as to:

(i) Resist the introduction of another network element to reduce excessive signalling,

(ii) Maximize the use of the existing WMN components and

(iii) Reduce the route processing unlike if it were different sets of elements.

The above conjectured framework is intended as a Layer 3 (IP layer) implementation based on the design assumptions of functional lower and upper layers mechanisms of the Internet Protocol layered model. This framework is informed by the need to provide seamless mobility support for mobile MC in WMN coupled with the fact that MAP is a mandatory hop in HMIPv $6^{26}$ and so is IGW in WMN and Core router in CBT. Hence IGW is to be referred to as Mobility Core Gateway Anchor Point (MOCGAP) - which consists of functionalities of internet gateway, Multicast Core router and hierarchical mobility management element.

\section{Load balancing technique design}

The provision of mobility mechanism on multicast enabled gateways may impact on the functions of IGW, however, the reduction in route processing among the network elements and reduction in the associated excessive signalling are positive gains. The load balancing technique is envisioned with some elements of redundancies incorporated into the hierarchical topology proposed. This will reduce the bottleneck or traffic concentration at MOCGAP and MR. For instance, multiple MOCGAP is assumed and the multicast packet being sent via unicast to nearest MOCGAP is to be replicated on all roots (Fig. 3). Load balancing is also incorporated to enhance the throughput of MR.

\section{Overall design}

In the proposed framework, the overall multicasting and mobility management Fig. 4 are specified thus:

(i) MAP of HMIPv6 occupies any level of the hierarchy however, IGW in WMN can only occupy the highest level hierarchy of the mesh router backbone. Hence MAP function in the proposed architecture is obtainable only at the topmost level of the WMN hierarchy.

(ii) The furthest MAP criterion in distance-based selection algorithm of HMIPv6 is envisioned to be modified in the proposed network architecture by 

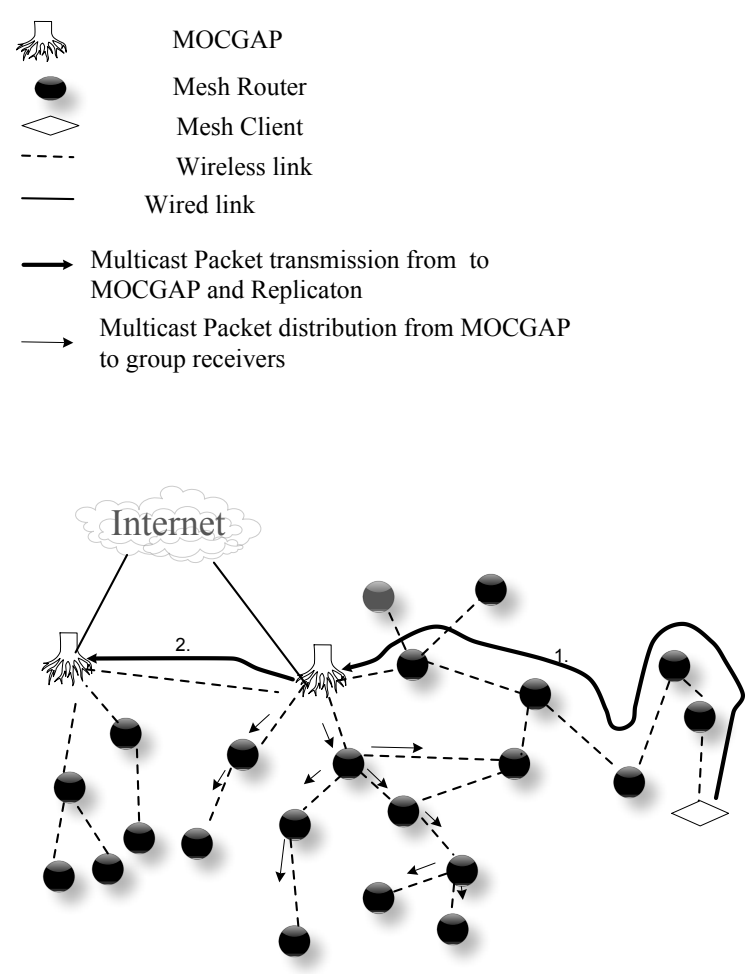

Fig. 3 Multicast packet replication and distribution to group receivers.

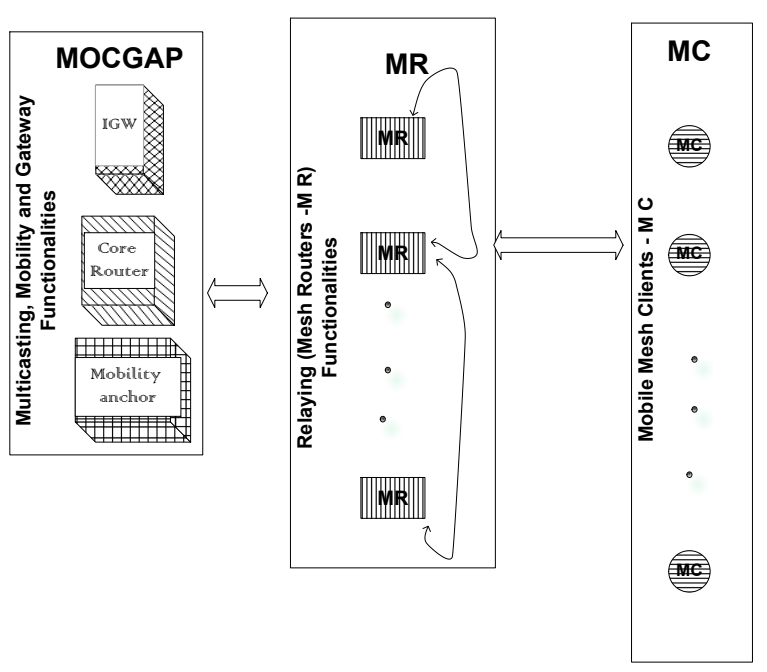

Fig. 4 Conceptual mobile multicast network architecture.

considering foremost the nearest MOCGAP to the mobile multicast sender, and load distribution on the MOCGAP.

(iii) IGW in addition to sending, receiving of IP datagram and translating IP destination addresses now define a regional address for the management

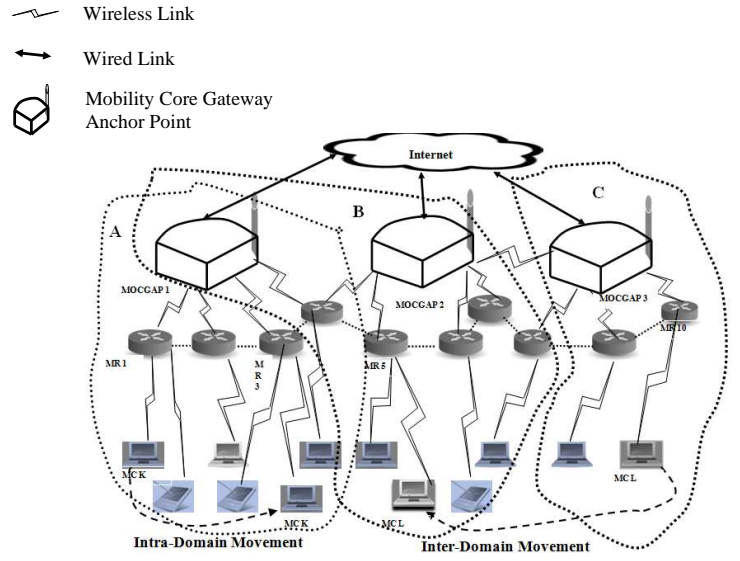

Fig. 5 Inter and intra domain handover in MOCGAP architecture.

of micro mobility within the proposed framework of the network architecture.

(iv) Load balancing techniques are envisioned for equipping multiple IGW cases to relieve congestion at MOCGAP and MR.

\section{MOBILE MULTICAST ROUTING}

The path selection metrics proposed in this framework is based on the objective of reducing delay of multicast packets in transit from the multicast sender to all receivers and resource consumption especially bandwidth. Hence nearness to group receivers of concern and the load on MOCGAP is given priority in the selection of root in the multiple MOCGAP implementations. In addition, since MOCGAP is optimally placed, the capacity violation of WMN components is mitigated in the CRP formulation. Fig. 5 shows the operational diagram for handover of two MC in two varying scenarios. The considerations in this case consist of three MOCGAP domains A, B and C, with each domain consisting of set of nodes vi's with at least one prominent MOCGAP, however MOCGAP domains can overlap as obtained for domains A and B, in which case, certain selection criteria are required.

Firstly, it depicts the movement of mesh client MCK from MR1 to MR3 classified as intra domain movement within sub domain A, hence (MOCGAP1) remains the same. Secondly, the movement of MCL from sub domain $\mathrm{C}$ to overlapping sub domains $\mathrm{A}$ and $\mathrm{B}$ consisting of MOCGAP1 and MOCGAP2 is a scenario of inter domain handover. The overlapping domains imply that the mobile $\mathrm{MC}$ is required to exploit extra functionality or priority ${ }^{21}$ for selecting from the two available MOCGAP. Apart from executing the selection procedure, the overall mobility and 
multicasting functionalities in the IGWs also involves series of node discovery, registration, and detection and so on. Handover delay is partitioned into mobile node registration delay (at mobility anchor) and binding update delay ${ }^{27}$. However, registration procedure consists of movement detection, router advertisement or solicitations and address configuration. For each undirected graph $\mathrm{G}(\mathrm{V}, \mathrm{E})$ modelling the WMN, V and $\mathrm{E}$ denotes the nodes and links, respectively, while the multicast service delivery is modelled as a tree. The proposed mobile multicast cost consists of queue length on MOCGAP, hop count or distance of the relay nodes and MOCGAP capacity. These can be expressed as weights on $\mathrm{V}$ and $\mathrm{E}$. The minimum/maximum cost is chosen based on the nature of objective function. Although collocating MAP with IGW that is enhanced with mobile multicast capability may constitute a single point failure, however, the selection procedure proposed in conjunction with the envisioned load balancing solution may address this problem especially in multiple gateway implementations.

\section{CONCLUSION AND FUTURE WORK}

The broadband capability and low installation cost offered by WMN amplifies its deployment rate especially in ad hoc environments involving group multimedia applications. These applications require multicasting and mobility management for effective delivery, however, the mandatory unicast protocol of IEEE 802.11s and disruption in internet session due to mobility reasons poses serious limitations in these cases. A structural design exploiting multicast architecture to solve mobile multicast problem is presented. This solution resists the introduction of another network component to avoid excessive signalling in the handshake routine. Thus a novel mobile multicast compliant architecture with custom multicasting and mobility management is proposed. The ongoing research works include the development of analytical model for the proposed network architectural framework.

\section{REFERENCES}

1. Shittu WA, Hashim AA, Anwar F, Kateeb WA (2008) A proposed QoS multicast routing framework for nextgeneration wireless mesh network. Int J Comp Sci Net Secur 8, 280-4.

2. Schmidt T, M, Waehlisch G, Fairhurst (2010) Multicast mobility in mobile IP Version 6 (MIPv6): problem statement and brief survey. IRTF RFC 5757.

3. Sanni ML, Hashim AA, Anwar F, Naji AW, Ahmed GSM (2011) Mobile multicast in wireless mesh networks. Aust J Basic Appl Sci 5, 957-66.
4. Xu Y, Qu R (2011) Solving multi-objective multicast routing problems by evolutionary multi-objective simulated annealing algorithms with variable neighbourhoods. J Oper Res Soc 62, 313-25.

5. Akyildiz I, Wang X, Wang W (2005) Wireless mesh networks: a survey. Comput Network 47, 445-87.

6. Xie J, Wang X (2008) A survey of mobility management in hybrid wireless mesh networks. IEEE Netw 22, 34-40.

7. Nguyen UT (2008) On multicast routing in wireless mesh networks. Comput Comm 31, 1385-99.

8. Bae S, Ko Y (2010) Efficient layer-2 multicasting for IEEE 802.11s based wireless mesh networks. In: Proc 2nd Int Conf on Ubiquitous and Future Networks (ICUFN), pp 109-14.

9. Tsai C, Yang C (2010) Design of the multicast service for mobile users in the 802.16 network environment. Comput Comm 3, 1030-48.

10. Murthy CSR, Manimaran G (2001) Resource Management in Real-Time Systems and Networks, MIT Press.

11. Zhang H, Yan Z, Gao S, Wang L, Wu Q, Li H (2010) Multicast source mobility support in PMIPv6 network. IETF Internet draft.

12. Deering S (1989) Host extensions for IP multicasting. IETF RFC 1112.

13. Sichitiu ML (2005) Wireless mesh networks: opportunities and challenges. Proc Wireless World Congress.

14. Amir Y, Danilov C, Hilsdale M, Musalou-Elefteri R, Rivera N (2006) Fast handoff for seamless wireless mesh networks. Proc ACM MobiSys'06, 83-95.

15. Na J-H, Park S, Moon J-M, Lee S, Lee E, Kim SH (2007) Roaming mechanism between PMIPv6 domains. IETF Internet Draft.

16. Al-Dubai AY, Zhao L, Zomaya A, Min G (2011) A novel multicast gateway based multi-hop routing for wireless mesh networks. Proc IEEE Int Conf on Communications.

17. Sanni ML, Hashim AA, Anwar F, Naji AW, Ahmed GSM (2012) Gateway placement optimisation problem for mobile multicast in wireless mesh networks. Proc 4th Int Conf on Computer and Communication Engineering (ICCCE'12), 446-451.

18. Ballardie A (1997) Core Based Tree (CBT) Multicast routing architecture. IETF RFC 2201.

19. Calvert KL, Zegura EW, Donahoo MJ (1995) Core selection methods for muticast routing. College of Computing, Georgia Institute of Technology, Atlanta, Georgia, GIT-CC-95/15.

20. Lin H, Lai SC (2000) A simple and effective core placement method for the core based tree multicast routing architecture. In: Proc IEEE Int Conf on Performance, Computing and Communications IPCCC 'OO, pp 215-9.

21. Lee S, Sahu S (2010) Core tree optimization in hybrid peer to peer real time broadcasting system. In: Proc IEEE Global Telecommunications Conference (GLOBECOM 2010) pp 1-5. 
22. Singh J, Veeraghavan P, Singh S (2004) Core based tree multicast (M-CBT) approach in supporting mobility. In: Proc IASTED Int Conference on Parallel and Distributed Computing and Networks, pp 147-52.

23. Helmy A (2002) Architectural framework for largescale multicast in mobile ad hoc networks. In: Proc IEEE Int Conf on Communications, pp 2036-42.

24. Soliman H, Castelluccia C, Malki K, Bellier L (2005) Hierarchical mobile IPv6 mobility management (HMIPv6). IETF RFC 4140.

25. Schmidt TC, Wahlisch M (2004) Performance analysis of multicast mobility in a hierarchical mobile IP proxy environment. Proc TERENA Conf Networking (TCN).

26. Cui X (2009) Mobility anchor point (MAP) reliability extension. IETF Internet draft.

27. Lei W, Landfeldt B (2006) On the problem of placing mobility anchor points in wireless mesh networks. In: Proc IEEE Workshop on Operator-Assisted (Wireless Mesh) Community Networks, pp 1-8. 